\title{
A novel mutation in DDR2 causing spondylo- meta-epiphyseal dysplasia with short limbs and abnormal calcifications (SMED-SL) results in defective intra-cellular trafficking
}

\author{
Adila Al-Kindi ${ }^{1 \dagger}$, Praseetha Kizhakkedath ${ }^{2 \dagger}$, Huifang Xu ${ }^{3}$, Anne John², Abeer Al Sayegh ${ }^{1}$, Anuradha Ganesh ${ }^{4}$,
} Maha Al-Awadi ${ }^{1}$, Lamya Al-Anbouri ${ }^{1}$, Lihadh Al-Gazali ${ }^{5}$, Birgit Leitinger ${ }^{3}$ and Bassam R Ali²

\begin{abstract}
Background: The rare autosomal genetic disorder, Spondylo-meta-epiphyseal dysplasia with short limbs and abnormal calcifications (SMED-SL), is reported to be caused by missense or splice site mutations in the human discoidin domain receptor 2 (DDR2) gene. Previously our group has established that trafficking defects and loss of ligand binding are the underlying cellular mechanisms of several SMED-SL causing mutations. Here we report the clinical characteristics of two siblings of consanguineous marriage with suspected SMED-SL and identification of a novel disease-causing mutation in the DDR2 gene.
\end{abstract}

Methods: Clinical evaluation and radiography were performed to evaluate the patients. All the coding exons and splice sites of the DDR2 gene were sequenced by Sanger sequencing. Subcellular localization of the mutated DDR2 protein was determined by confocal microscopy, deglycosylation assay and Western blotting. DDR2 activity was measured by collagen activation and Western analysis.

Results: In addition to the typical features of SMED-SL, one of the patients has an eye phenotype including visual impairment due to optic atrophy. DNA sequencing revealed a novel homozygous dinucleotide deletion mutation (c.2468_2469delCT) on exon 18 of the DDR2 gene in both patients. The mutation resulted in a frameshift leading to an amino acid change at position $\mathrm{S} 823$ and a predicted premature termination of translation (p.S823Cfs*2). Subcellular localization of the mutant protein was analyzed in mammalian cell lines, and it was found to be largely retained in the endoplasmic reticulum (ER), which was further supported by its N-glycosylation profile. In keeping with its cellular mis-localization, the mutant protein was found to be deficient in collagen-induced receptor activation, suggesting protein trafficking defects as the major cellular mechanism underlying the loss of DDR2 function in our patients.

Conclusions: Our results indicate that the novel mutation results in defective trafficking of the DDR2 protein leading to loss of function and disease. This confirms our previous findings that DDR2 missense mutations occurring at the kinase domain result in retention of the mutant protein in the ER.

Keywords: DDR2, Spondylo-meta-epiphyseal dysplasia, Trafficking defect, SMED-SL, ERAD, Optic atrophy

\footnotetext{
* Correspondence: bassam.ali@uaeu.ac.ae

${ }^{\dagger}$ Equal contributors

${ }^{2}$ Department of Pathology, College of Medicine and Heath Sciences, United

Arab Emirates University, P.O. Box 17666, Al Ain, United Arab Emirates

Full list of author information is available at the end of the article
} 


\section{Background}

Spondylo-meta-epiphyseal dysplasia (SMED), short limbhand type (SMED-SL, OMIM 271665) is a rare autosomal recessive disorder affecting human skeletal growth. The condition is characterized by disproportionately short stature, platispondyly, abnormal epiphyses and metaphyses, shortening of the lower and upper limbs, short broad fingers and punctate calcifications [1-4]. This bone dysplasia is progressive, with serious complications leading to death in some cases. Atlantoaxial instability resulting in cord damage has been the most reported cause of death $[2,5]$. Homozygous mutations occurring in the Discoidin domain receptor 2 gene (DDR2, MIM 191311) have been identified as the cause for this severely dwarfing condition $[6,7]$. The DDR2 gene encodes one of the two members of a unique receptor tyrosine kinase (RTK) subfamily known as the discoidin domain containing receptors (DDRs), which recognize collagen as their ligands $[8,9]$. Upon collagen binding, the receptor displays delayed and sustained tyrosine phosphorylation which further elicits downstream signalling to cellular metabolic pathways that cross-talk at various points $[10,11]$. In addition, dysregulation of DDR2 has been shown to be associated with various human diseases such as fibrosis, arthritis and cancer and like other RTKs, DDRs are emerging as potential therapeutic targets [12].

DDR2 is commonly expressed in cells of mesenchymal origin and is activated by fibrillar collagens $[8,9]$ and collagen X [13]. DDR2 has been shown to play a critical role in cell invasion and collagen remodelling through the regulation of matrix metalloproteases and collagen fibrillogenesis [10,14-16]. The involvement of DDR2 in skeletal growth was demonstrated by the DDR2 knockout mice, which display skeletal abnormalities that reflect the SMED-SL phenotype of humans [17]. The abnormal skeletal development in DDR2 deficient mice was due to reduced chondrocyte proliferation in the growth plate. A spontaneous, autosomal recessive mutation in a mouse colony was characterised that resulted from deletion of most of the $D d r 2$ gene [18]. These mutant mice were also found to be infertile due to gonadal dysfunction including impaired spermatogenesis and ovulation, which were attributed to defects in overall endocrine function [18-20].

DDRs are plasma membrane RTKs comprising an extracellular domain (ECD), a transmembrane domain (TM), a large cytosolic juxtamembrane domain and a Cterminal catalytic tyrosine kinase domain. The ECD is necessary and sufficient for ligand binding and consists of an $\mathrm{N}$-terminal discoidin homology (DS) domain followed by a region unique to the DDRs that contains a DS-like domain [21-23]. The DDRs exist as preformed homodimers on the cell membrane even in the absence of collagen $[24,25]$. The exact signaling pathways and binding partners through which DDR2 controls bone growth remain unknown despite significant progress made in recent years in understanding the structural basis of collagen recognition [26]. In recent reports it was shown that DDR2 modulates the phosphorylation of Runx2, a master transcription factor involved in skeletal development $[27,28]$. In addition, DDR2 has been shown to mediate the secretion of lysyl oxidase [29], an enzyme that catalyzes cross-linking of collagen fibers, an essential modification to strengthen bone.

A recent study has shown that the DDR2 ECD aids the formation of mineralized calcium deposits in vitro, and this activity is independent of the tyrosine kinase activity [16]. The authors proposed that the presence of DDR2 ECD in the mutant proteins along with impaired signaling leading to increased calcification could be a potential mechanism in SMED-SL. Mutations prevalent in SMED-SL disorder have been mapped to the extracellular domain and tyrosine kinase domain of DDR2 [6,7]. Recently we have shown that DDR2 missense mutations occurring at the kinase domain (p.T713I, p.I176R, p. $\mathrm{R} 752 \mathrm{C})$ result in retention of the mutant protein in the endoplasmic reticulum (ER), while the ectodomain mutant (p.E113K) is expressed on the cell surface, but failed to bind to collagen, thus elucidating two different cellular mechanisms resulting in loss of function of the protein leading to disease [7].

ER retention of misfolded proteins and subsequent degradation (ERAD) is a recurring theme in many skeletal disorders [30-33] and numerous other monogenic diseases [31,34-36]. Inhibition of ERAD coupled with proteostasis modulation has been shown to enhance folding, trafficking and activity of unstable proteins and is emerging as a potential strategy to combat many protein misfolding disorders [37]. Defining the potential contribution of specific mutations and intracellular stress to the pathophysiology of the disease will pave avenues for targeted therapeutic intervention and disease management in the future.

We report here the clinical and molecular findings in SMED-SL patients of consanguineous origin from Oman and the identification of a novel disease-causing truncating mutation in DDR2. The sub-cellular localization and functional status of the mutant and wild type proteins were compared in a mammalian expression system. Our results indicate that the novel mutation results in defective trafficking of the protein and loss of its activation by collagen.

\section{Methods}

\section{Clinical evaluations}

This study complies with the Helsinki Declaration and has been approved by Al-Ain District Human Research Ethics Committees (protocol No. AAMD/HREC 10/09) and the family provided an informed consent. Clinical assessment 
was performed by a clinical geneticist and the radiography imaging of the subjects was also evaluated.

\section{Chemicals and reagents}

Collagen I (acid-soluble from rat tail) was from Sigma (Gillingham, UK). The antibodies and their sources were as follows: antibodies for immunofluorescence: mouse anti-HA-tag monoclonal antibody (dilution 1:200; Cell Signaling Technology), rabbit anti-calnexin polyclonal antibody (dilution 1:200, Santa Cruz), Alexa Fluor 568-goat anti-mouse IgG (dilution 1:200; Molecular Probes), Alexa Fluor 568-goat anti-rabbit IgG (dilution 1:200; Molecular Probes), Alexa Fluor 488-goat anti mouse IgG (dilution 1:200, Molecular Probes). Antibodies for Western blotting: goat anti-DDR2 from R \& D Systems (Abingdon, UK); mouse anti-phosphotyrosine, clone 4G10, from Upstate Biotechnology (Lake Placid, NY); sheep anti-mouse Ig-horseradish peroxidase (Amersham Biosciences UK, Chalfont St Giles, UK); rabbit anti-goat Ig-horseradish peroxidase (Zymed Laboratories, San Francisco, CA).

\section{Mutation screening}

Genomic DNA was extracted from peripheral leukocytes using the Flexigene DNA kit (Qiagen). The coding exons and exon-flanking sequences were analyzed by PCR (Taq polymerase, Qiagen) followed by direct sequencing using the BigDye Terminator v3.1 Cycle Sequencing kit (Applied Biosystems, Foster City, CA). Capillary electrophoresis was performed on an ABI PRISM 3130xl DNA Analyzer (Applied Biosystems). Primers and PCR conditions for amplification of the coding exons (Exons 3-18) and splice sites have been described previously [7]. Sequences were aligned to the NCBI reference sequence NM_001014796, using ClustalW2 algorithm and mutations were designated with reference to the translation start site.

\section{Generation of mammalian expression constructs}

The frame-shift mutation, c.2468_2469delCT was introduced into the untagged expression vector, pcDNA3.1DDR2 [22] by site directed mutagenesis using Pfu Ultra HF polymerase (Stratagene, La Jolla, CA). The primers used were: DDR2-S823Cfs"-F: CATTTGTCCTGACTGTGTA TAAGCTGATG, DDR2-S823Cfs*-R: CATCAGCTTATA CACAGTCAGGACAAATG. This construct was subsequently used for performing deglycosylation and receptor binding assays. An HA tag was inserted prior to the stop codon (introduced by the frame-shift mutation) of the mutated construct, using two cycles of site directed mutagenesis. In the first cycle, the initial five amino acids (YPYDV) of the HA tag were introduced at the C-terminus of the mutated DDR2, using the primers: DDR2_Mut2A_FP: GTCCTGACTGTGTATACCCATA CGATGTTTAAGCTGATGCTCAGCTG, DDR2_Mut2A_RP: CAGCTGAGCATCAGCTTAAACATCGTAT
GGGTATACACAGTCAGGAC. The remaining four amino acids (PDYA) of the tag were introduced to the above construct using the following primers: DDR2 Mut2B_FP: ATACCCATACGATGTTCCAGATTACGCT TAAGCTGATGCTCAGC, DDR2_Mut2B_RP: GCTGAG CATCAGCTTAAGCGTAATCTGGAACATCGTATGGG TAT. All the plasmids have been sequenced to confirm the introduction of intended changes.

\section{Cell culture and transfection}

HeLa cells were cultured in DMEM (Invitrogen, Carlsbad, CA) supplemented with $10 \%$ fetal bovine serum, $2 \mathrm{mM} \mathrm{L-}$ glutamine and $100 \mathrm{U} / \mathrm{ml}$ penicillin/streptomycin at $37^{\circ} \mathrm{C}$ with 5\% CO2. For localization experiments, cells were grown on sterile cover slips in 24-well tissue culture plates and transient transfection was performed by FuGENE HD transfection reagent according to the manufacturer's instructions (Promega, Madison, WI) using $0.5 \mu \mathrm{g}$ plasmid DNA. GFP-H-Ras plasmid was used as a plasma membrane marker and co-transfected with HA-tagged wild type or mutant plasmids. The cells were processed for staining and imaging after 24 hours of transfection.

Human embryonic kidney (HEK-293) cells (ATCC, Manassas, VA, USA) were cultured in Dulbecco's modified Eagle's medium/F12 medium (Invitrogen) supplemented with 10\% fetal bovine serum (Invitrogen), penicillin (10 U/ $\mathrm{ml})$ and streptomycin $(100 \mu \mathrm{g} / \mathrm{ml})$ at $37^{\circ} \mathrm{C}$ with $5 \% \mathrm{CO} 2$. For transfection, cells were grown in 24-well tissue culture plates and were transfected with untagged DDR2 wild-type or mutant plasmid DNA using calcium phosphate precipitation.

\section{Immunocytochemistry and imaging}

Twenty-four hours after transfection, HeLa cells grown on cover slips were washed with phosphate-buffered saline (PBS) and fixed by methanol at $22^{\circ} \mathrm{C}$ for $5 \mathrm{~min}$. Fixed cells were washed in PBS three times and blocking was carried out in 1\% BSA (Sigma) in PBS for $30 \mathrm{~min}$ at room temperature. After blocking, the cells were incubated with either mouse monoclonal anti-HA antibody alone or co-stained with both mouse monoclonal anti-HA antibody and rabbit polyclonal anti-calnexin antibodies, for $45 \mathrm{~min}$ at room temperature. The cells were washed with PBS, and incubated with the respective secondary antibodies for $45 \mathrm{~min}$ at room temperature, washed several times with PBS and mounted in immunofluor medium (ICN Biomedicals). Confocal microscopy and imaging was performed with a Nikon Eclipse system (Nikon Instruments Inc., Melville, NY).

\section{Collagen-induced DDR2 autophosphorylation}

The assay was performed as described previously in details [22]. Twenty-four hours after transfection, HEK-293 cells were incubated with serum-free medium for $16 \mathrm{~h}$. 
Cells were then stimulated with collagen I $(5-50 \mu \mathrm{g} / \mathrm{ml})$ for $90 \mathrm{~min}$ at $37^{\circ} \mathrm{C}$. Cells were lysed in $1 \%$ Nonidet P-40, $150 \mathrm{mM} \mathrm{NaCl}, 50 \mathrm{mM}$ Tris, pH 7.4, 1 mM EDTA, $1 \mathrm{mM}$ phenylmethylsulfonyl fluoride, $50 \mu \mathrm{g} / \mathrm{ml}$ aprotinin, $1 \mathrm{mM}$ sodium orthovanadate and $5 \mathrm{mM} \mathrm{NaF}$. Aliquots of the lysates were analyzed by SDS-PAGE followed by blotting onto nitrocellulose membranes. The duplicate blots were probed with either anti-phosphotyrosine monoclonal antibody or anti-DDR2 antibodies followed by corresponding horseradish peroxidise-conjugated secondary antibodies. Detection was performed using Pierce ECL 2 Western Blotting substrate (Thermo Scientific) and an Ettan DIGE Imager (GE Healthcare Biosciences).

\section{Endoglycosidase $\mathrm{H}$ deglycosylation assay}

Forty eight hours after transfection, HEK-293 cells were lysed as described above. Twenty microliter of each cell lysate was denatured in $1 \times$ glycoprotein denaturing buffer $(0.5 \%$ SDS and $1 \% \beta$-mercaptoethanol) for $5 \mathrm{~min}$ at $100^{\circ} \mathrm{C}$. The denatured lysates were then split into two equal aliquots which were incubated for $3 \mathrm{~h}$ at $37^{\circ} \mathrm{C}$ in the presence or absence of $10 \mathrm{U}$ of Endoglycosidase $\mathrm{H}$ (New England Biolabs). Samples were then resolved on 7.5\% SDS-PAGE followed by blotting onto nitrocellulose membranes and probing with anti-DDR2 antibodies, as described above.

\section{Results}

\section{Clinical characteristics of SMED-SL patients}

The parents are first cousins of Omani origin (Figure 1A). The two affected children, a boy and a girl, were assessed, aged 10 years and 7 years. Both presented with severe short stature, height well below $3^{\text {rd }}$ centile $(-6 \mathrm{SD}-7 \mathrm{SD})$ with relative macrocephaly, wide anterior fontanelle and partial alopecia at the vertex. There was shortening of all limbs, particularly the distal segments, madelung deformity of the forearm with limited extension of the elbow joints, and bowing of the tibias. The hands and feet were very small with short stubby fingers and toes with hypoplastic nails (Figure 1B panels (b) and (c)). The chest was narrow with pectus excavatum and there was thoracic scoliosis with lumber gibbus. The children had coarse facial features with midface hypoplasia, hypertelorism, infraoribital fullness with creases, short flat nose with wide anteverted nares, long philtrum with prominent full lower lip, misaligned teeth and micrognathia. Both had moderate conductive hearing loss due to glue ear. The girl had progressive visual impairment due to bilateral optic atrophy. Both children have normal intelligence. Both parents, maternal and paternal grandmother are said to be short but no measurements were available.

Skeletal survey on both children revealed changes typical of SMED-SL including short and broad long bones with irregular, flared metaphyses of the proximal and distal ends with irregular trabecular structure (Figure 1B-f). In the spine there was platyspondyly, pear shaped vertebrae, anterior beaking, irregular endplates and punctate calcification (Figure 1B-e). There was a small thoracic diameter with short broad ribs. The tubular bones of the hands were very short and broad with diaphyseal constriction (Figure 1B-f). The metacarpal bones were narrower at the proximal than distal ends giving the appearance of drumsticks and the distal phalanges were triangular in shape (Figure 1B-d).

\section{The patients have a homozygous truncating mutation in the DDR2 gene}

The genomic DNA from the parents and the male patient was isolated and used as template to amplify all the DDR2 gene coding exons and splice sites. Direct sequence analysis of the PCR amplicons revealed a homozygous two nucleotide deletion in exon 18 (c.2468_2469delCT) of the patient (Figure 2A). In addition to causing an amino acid substitution, S823C, the deletion leads to a frame-shift followed by a stop codon two amino acid residues downstream of the mutation (p.S823Cfs*2). Both parents were found to be heterozygous for the mutation (Figure $2 \mathrm{~A}$ ). The mutation is predicted to result in a truncated DDR2 protein lacking the C-terminal 32 amino acids of the cytoplasmic tyrosine kinase domain. The serine residue at position 823 and the amino acid residues in the truncated region of the mutant are highly conserved across various species (Figure 2B). This mutation has not been previously reported in any of the SNP or clinical variant databases. Mutation prediction programmes (Mutation taster) predicted that these changes are highly likely disease-causing by affecting the protein structure and/or function. Additionally, truncating mutations occurring in the last exon of a gene are not suppressed by nonsense mediated mRNA decay (NMD) and might exert a dominant negative effect [38].

\section{The mutation leads to defective intracellular targeting of the mutant protein}

Previously we reported that missense mutations located on the intracellular kinase domain of DDR2 resulted in the ER retention and loss of activation of the protein. To investigate the implications of the novel disease-causing mutation (S823Cfs*) on the DDR2 protein, we generated by site directed mutagenesis, the mutant cDNA fused to an HA epitope tag at the C-terminus and expressed it in a mammalian expression system. The subcellular localization of the mutant protein was determined by immunohistochemical staining and confocal imaging of HeLa cells expressing the mutant protein. As expected, the wild type DDR2 was found to localize primarily to the plasma membrane and co-localize with the plasma membrane protein $\mathrm{H}$-Ras tagged with GFP (Figure 3, panels A,B and C). On the contrary, the localization pattern of the $\mathrm{S} 823 \mathrm{Cfs}$ * 

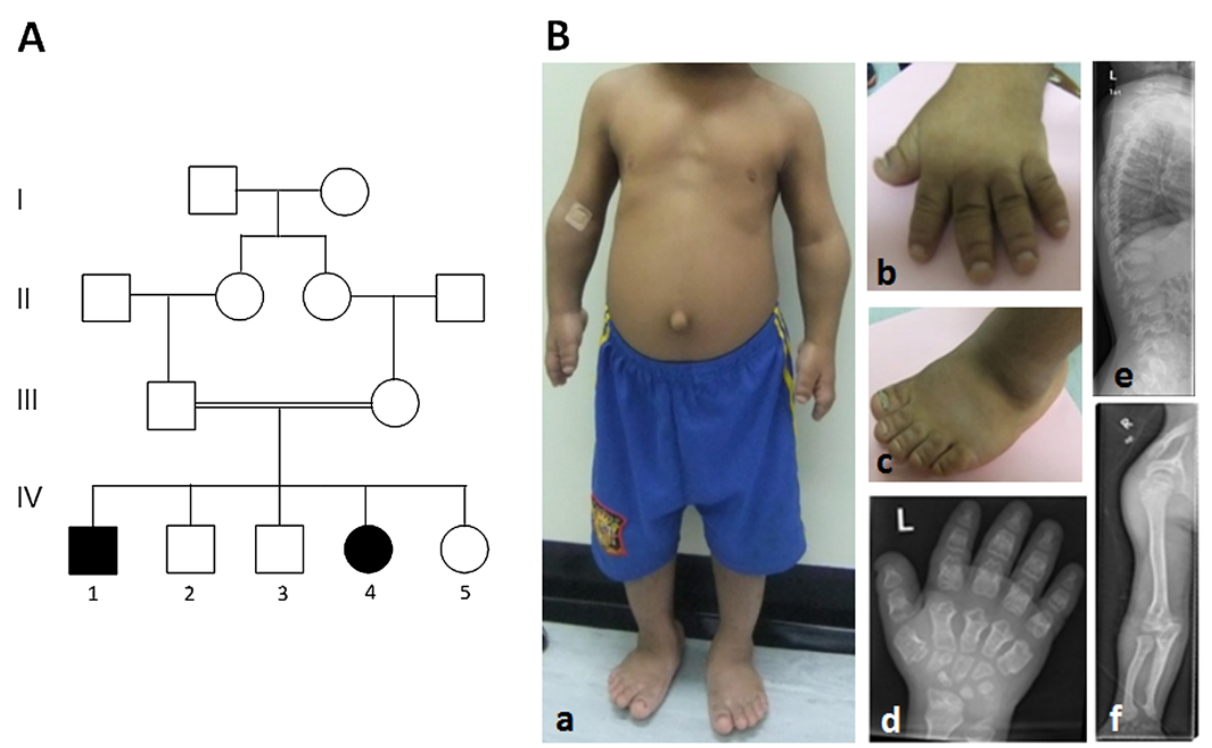

Figure 1 Pedigree, dysmorphic features and radiographic findings in the affected family. (A) Pedigree of the consanguineous Omani family. The family displays typical recessive modes of inheritance. Double bar represents consanguinity. Affected members are represented by filled symbols. (B) Dysmorphic features (a) 10 year old affected boy, note short limbs, narrow chest and protuberant abdomen with everted umbilicus (b) \& (c) short broad hands and feet with stubby fingers and toes with short hypoplastic nails (d) X-ray of the left hand at 10 years of age showing short and broad tubular bones with diaphyseal constriction, narrowing of the metacarpal bones proximally, triangular distal phallanges, and irregular epiphyses which are cone shaped. $L$ on the upper left corner of the X-ray refers to left hand (e) Lateral X-ray of the spine showing platyspondyly with pear shaped vertebrae, some showing anterior beaking, irregularities of the vertebral endplated and punctate calcifications (f) X-ray of the upper arm at 10 years of age showing mesomelic shortening of the long bones, madelung deformity, and irregular distal epiphyses of radius and ulna.

mutant was distinct from that of the wild type protein. As shown in Figure 3 (panels D,E and F), the mutant protein was found to localize predominantly in the endoplasmic reticulum, along with calnexin. A fraction of the wild type protein was also found to co-localize with the ER marker calnexin (Figure 3, panels G,H and I); this is presumably the in transit biosynthetic fraction of the protein or the fraction that failed the stringent ER quality control machinery. In cells co-transfected with GFP-H-Ras and the S823Cfs* mutant, the fluorescence from the mutant was found to be excluded from the plasma membrane (Figure 3, panels J,K and L).

\section{Incomplete glycosylation and loss of activation of the disease-causing mutant}

In all the previously reported SMED-SL missense mutants of DDR2, collagen mediated activation was disrupted due to either mis-folding and trafficking defects or loss of ligand binding. The new disease-causing mutation in this report is predicted to result in a truncated protein which lacks the last 32 amino acid residues of the cytoplasmic tyrosine kinase domain. To determine whether the novel disease-causing mutant was functionally active in vivo, we expressed the mutant and wild-type DDR2 in HEK-293 cells and analyzed their N-glycosylation status and activation by collagen.
Due to glycosylation, DDR2 is often detected as a mixture of $3-4$ bands between $125 \mathrm{kDa}$ and $130 \mathrm{kDa}$ $[22,39,40]$, with the higher molecular weight forms representing heavily glycosylated mature protein and the smaller molecular weight bands corresponding to immature, partially glycosylated forms. In Western blot of lysates from HEK-293 cells overexpressing the S823Cfs* mutant, only the lowest molecular weight form was detected which was also found to migrate faster than the corresponding band in the wild type (Figure 4A, lower panel). The highest and intermediate molecular weight forms were not observed for the mutant while the wild type DDR2 was detected as a mixture of three bands including the highest and intermediate molecular weight forms. Based on previous reports, the lower molecular weight form of the mutant is presumably the ER retained partially glycosylated folding intermediate. This was further supported by deglycosylation of the mutant protein with Endoglycosidase $\mathrm{H}$ (Endo $\mathrm{H}$ ), which specifically removes oligosaccharides of the high mannose and hybrid (pre-Golgi) forms, but not complex carbohydrate structures attained in the Golgi. Figure $4 \mathrm{~B}$ shows the Endo $\mathrm{H}$ treated protein extracts from wild type and mutant over-expressing cells. The mutant protein was found to be sensitive to Endo $\mathrm{H}$ as was the lowest molecular weight wild type form. Deglycosylation caused both the forms to resolve as lower molecular weight bands. The higher molecular weight 
A

c.2468_2469delCT (p.S823Cfs*2)
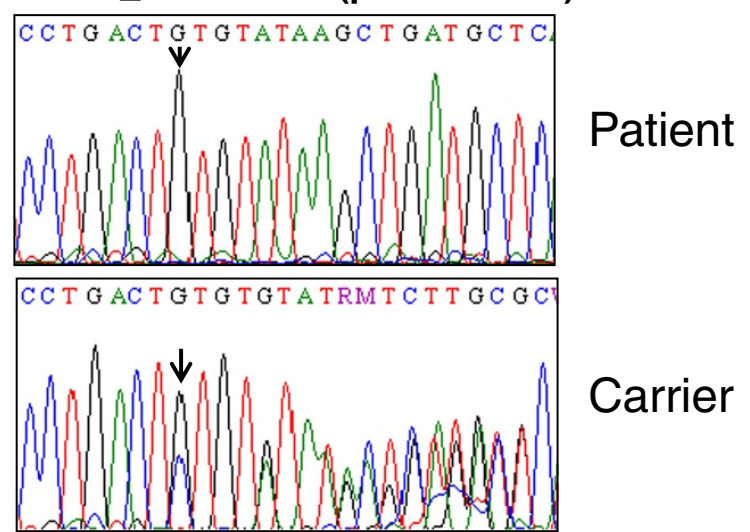

\section{Carrier}

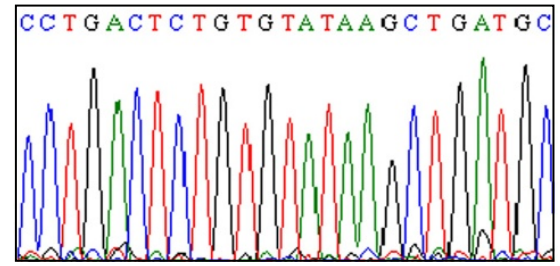

\section{Normal}

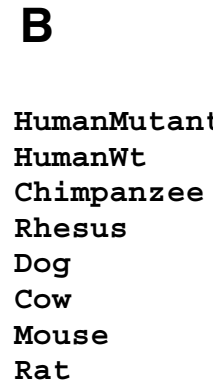

Ser 823

$\downarrow$

RDQGRQTYLPQPAICPDCV

RDQGRQTYLPQPAICPD SVYKLMLSCWRRDTKNRP SFQEIHLLLLQQGDE

RDQGRQTYLPQPAICPDSVYKLMLSCWRRDTKNRPSFQEIHLLLLQQGDE

RDOGROTYLPOPAICPD SVYKLML SCWRRDTKNRP SFOEIHLLLLOQGDE RDQGRQIYLPQPVICPD SVYKLMLSCWRRDTKHRP SFQEIHLLLLQQGDE RDQGRQTYLPQPAICPDSVYKLMLSCWRRDTKHRP SFQEIHLLLLQQVDD RDQGRQIYLPQPALCPD SVYKLMLSCWRRETKHRP SFOEIHLLLLQQGAE RDQGRQIYLPQPAVCPD SVYKLMLSCWRRETKHRP SFQEIHLLLLQQGAE

Figure 2 Identification of a novel dinucleotide deletion in the SMED-SL patient. Direct sequencing analysis was carried out for all the coding exons of the DDR2 gene. (A) This panel shows a representative chromatogram of the DNA sequences indicating the C.2468_2469delCT mutation in the affected individuals and their parents. Arrows indicate the position of the C.2468_2469delCT mutation in the patient and one of the heterozygous carriers (B) Multiple sequence alignment of mutant and wild type DDR2 proteins from different species. The S823 residue is highlighted.

forms of the wild type DDR2 were found resistant to Endo $\mathrm{H}$ treatment. This confirms the ER localization of the mutant which was also evident from the confocal images (Figure 3).

Only the completely glycosylated and mature forms of DDR2 of the highest molecular weight $(130 \mathrm{kDa})$ is reported to reach the cell surface and get activated by collagen $[22,39,40]$, which is a prerequisite for DDR2 receptor-mediated signaling. Upon collagen binding, DDR2 undergoes autophosphorylation at tyrosine residues in the cytoplasmic domain. HEK-293 cells expressing wild type or mutant DDR2 were treated with collagen as described in the Methods section, and tyrosine phosphorylation was analyzed by Western blotting using anti-phosphotyrosine (PY) antibodies. In contrast to wild type DDR2, which, as expected, showed a strong collagen-induced phosphorylation signal, no phosphorylation signal was detected for the S823Cfs" mutant (Figure 4A). These data strongly suggest that the mutation has led to loss of the DDR2 protein in vivo function.

\section{Discussion}

We report here the clinical findings and radiological features of two siblings with SMED-SL and a novel DDR2 homozygous mutation. In addition to exhibiting the typical clinical and radiological features consistent with SMED-SL, our patients had some phenotypic variations between them. Progressive visual impairment and bilateral optic atrophy were observed in one of the patients. Abnormal eye movements and bilateral optic atrophy 

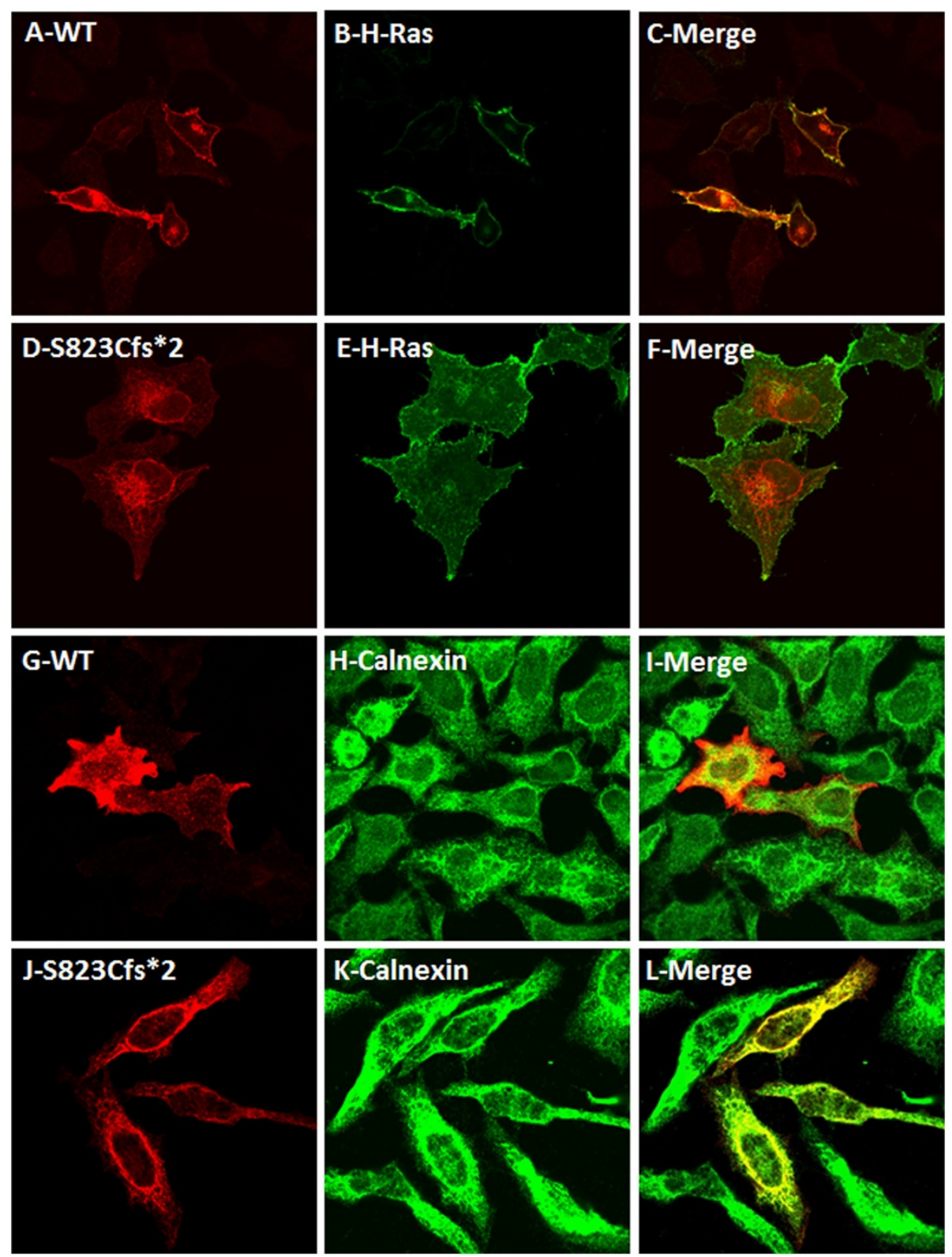

Figure 3 Comparison of intracellular localization of DDR2 wild type and S823Cfs* mutant in relation with plasma membrane marker H-Ras and ER marker calnexin. HeLa cells were co-transfected with C-terminally HA-tagged wild type or mutant DDR2 and EGFP tagged H-Ras constructs and stained with anti-HA antibodies. (A) and (D) shows the localization patterns of WT and the mutant DDR2 proteins. (B) and (E) shows the localization of the marker protein H-Ras to the plasma membrane. (C) shows co-localization of WT-DDR2 with H-Ras and (F) shows exclusion of S823Cfs*-DDR2 from the plasma membrane. (G) and $(\boldsymbol{J})$ show distribution of HA-tagged wild type DDR2 and S823cfs* mutant in HeLa cells transfected with the indicated construct. $\mathbf{( H )}$ and $\mathbf{( K )}$ show the localization of the ER marker protein calnexin. (I) and $\mathbf{( L )}$ show the extent of co-localization of DDR2 proteins with calnexin. For presentation purpose, images (G)-(L) were pseudocolored as either red (DDR2) or green (calnexin) using ImageJ software.

had been described before in SMED-SL [4]. The eye phenotype could be attributed to the fact that DDR2 is a plasma membrane RTK that functions as a collagen receptor to collagen II, a fibrillar collagen found in cartilage and the vitreous humor of the eye. Importantly the eye is made up of $80 \%$ collagen. Although the involvement of DDR2 in pathophysiologies related to the eye is not characterized, there is molecular evidence that
DDR1 and DDR2 mRNAs and proteins are expressed in corneal epithelial cells, keratocytes and endothelial cells [41].

The disease-causing frame-shift mutation reported here is located on exon 18 of the DDR2 gene, which is the last coding exon. The frame-shift causes an amino acid change of S823C and premature termination of translation, two amino acid residues downstream of the 

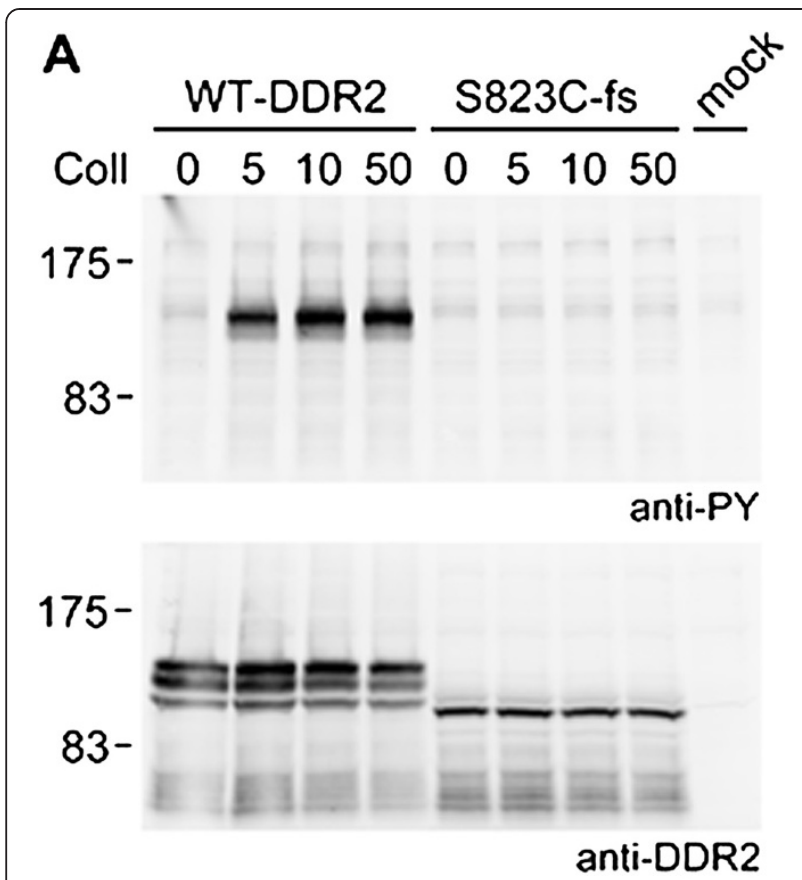

B

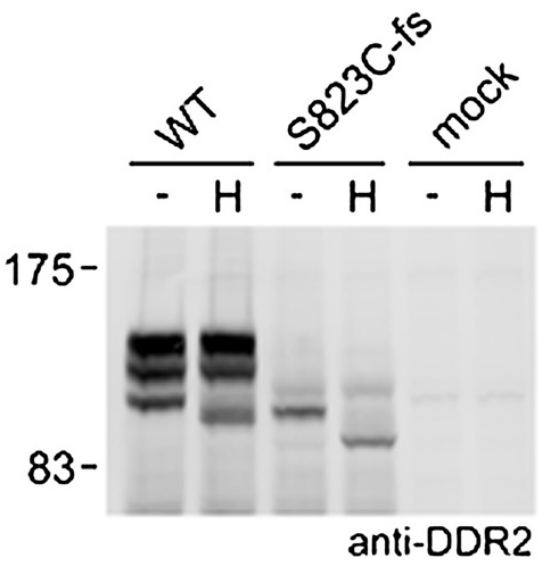

Figure 4 Defective cellular trafficking causes loss of collageninduced signalling for the DDR2-S823Cfs* mutant. Full-length untagged wild-type DDR2 or S823Cfs* mutant were transiently expressed in HEK-293 cells. (A) Cells were stimulated for $90 \mathrm{~min}$ at $37^{\circ} \mathrm{C}$ with rat tail collagen I at the indicated concentrations (in $\mu \mathrm{g} / \mathrm{ml}$ ). Cell lysates were analyzed by SDS-PAGE and Western blotting. The blots were probed with anti-phosphotyrosine (anti-PY) monoclonal antibody 4G10 (upper blot) or polyclonal anti-DDR2 antibodies (lower blot). (B) Cell lysates were treated with Endoglycosidase $\mathrm{H}(\mathrm{H})$ or left untreated (-) for $3 \mathrm{~h}$ at $37^{\circ} \mathrm{C}$ and analyzed by SDS-PAGE and Western blotting. The blot was probed with polyclonal anti-DDR2 antibodies. The positions of molecular markers (in $\mathrm{kDa}$ ) are indicated. The experiments were carried out twice with very similar results.

site of mutation. Premature termination codons appearing at the last coding exons of genes are found to be clinically relevant in several monogenic diseases. Transcripts of genes carrying premature termination codons located at least 50 nucleotides upstream of the last intron, are targets of an
mRNA surveillance mechanism known as nonsense mediated decay (NMD). But mRNAs carrying the mutation in the last exon escape this mechanism which results in translation of truncated proteins which may exert a dominant negative effect even in the heterozygous carriers of the mutant allele [38]. Recently it was shown that Hajdu-Cheney syndrome, a rare skeletal disorder, is caused by heterozygous mutations clustering to the last exon of the NOTCH 2 gene, which code for a truncated protein that acts in a gain-of-function manner [42]. In a frame-shift mutation reported in DDR1 gene, DDR1d, which is predicted to result in a membrane anchored, kinase deficient receptor, the mutant did not exert a dominant negative inhibition on the normal allele [43]. To establish a dominant negative effect for the novel DDR2 mutant, more detailed clinical, molecular and cellular analysis of the affected family is required.

The disease-causing mutation affects amino acid residues in the tyrosine kinase domain of DDR2. All kinases adopt a very similar structure [44] and hence loss of 32 amino acids from the DDR2 C-terminus is likely to affect protein stability, folding and plasma membrane targeting. Most kinases have a C-terminal tail, but in the case of DDR1 and DDR2, the catalytic kinase domains are followed by very short C-terminal tails. A crystal structure of the kinase domain of DDR1 shows that the C-terminal amino acids that are commonly assigned to a C-terminal 'tail' are actually part of an $\alpha$ helix in DDR1, which tightly interacts with the rest of the domain [45]. Therefore in the highly similar DDR2, loss of the 32 amino acids from the tightly folded C-terminus is likely to cause misfolding of the kinase domain and subsequent retention in the ER. Moreover, the high molecular weight form of DDR2 has been reported to be inherently unstable and dependent largely on glycosylation for efficient trafficking to the cell membrane [40]. We show here that the $\mathrm{S} 823 \mathrm{Cfs}^{*}$ mutant is retained in the ER and fails to reach the plasma membrane. As expected, the wild type protein localized predominantly to the plasma membrane, and a fraction of the wild type protein was observed also in the ER. Biochemical analysis of the mutant and wild type protein confirmed their intracellular distribution. The predominant form of mutant protein expressed in cells is found to be the immature lower molecular weight forms characteristic of ER retained folding intermediates. The wild type DDR2 exists as a mixture of mature high molecular weight and lower molecular weight intermediate forms. Further, the low molecular weight Endo H sensitive S823Cfs* mutant was not phosphorylated in response to collagen activation. Only the cell surface expressed DDR receptors bind to collagen, which induces phosphorylation of tyrosine residues in the cytoplasmic domain and downstream signalling [11]. Our results indicate that the S823Cfs* mutation results in an ER retained protein which is inactive in vivo upon adding collagen to the 
culture media. This is in agreement with our previous findings that mutations affecting the kinase domain of DDR2 result in misfolding and defective trafficking of the protein [7]. Our results also indicate that as predicted from the crystal structure of DDR1, the C-terminal amino acids are crucial for the stability of DDR2.

We have elucidated previously that missense mutations occurring in DDR2 cause loss of function of the protein through two mechanisms, namely retention of the mutant proteins in the ER and loss of ligand binding activity [7]. The ER has a sophisticated quality control system that ensures trafficking of only mature proteins in their native conformation. Misfolded proteins that fail to conform to the ER quality control are retained in the ER and subsequently targeted for degradation by the ubiquitin/proteasomal machinery and have been implicated in the pathogenicity of many congenital disorders [31]. Recently it has been shown in a transgenic mouse model of growth plate dysplasia, that chronic ER stress is sufficient to cause decreased chondrocyte proliferation and reduction in bone growth, without inducing any alterations to the architecture and organization of cartilage extracellular matrix [30]. Chronic ER stress due to persistent accumulation of misfolded proteins has also been proposed to be involved in the development of other conditions including diabetes in some cystic fibrosis patients [46]. Concurrent to this hypothesis, in SMED-SL patients carrying DDR2 mutations encoding plasma membrane expressed variants, the clinical symptoms were reported to be less severe than in patients carrying DDR2 mutations encoding ER retained variants [7].

Spondylo-meta-epiphyseal dysplasia is a severe form of dwarfism with serious complications and for which no effective treatment is available. A previous study [5] suggested that SMED-SL should be included in the list of genetic disorders causing death. In genetic diseases caused by the impaired function of a single gene, various interconnected pathways are affected, making the identification of relevant pharmaceutical targets difficult. Accurately defining the consequences of underlying mutations is a challenge and prerequisite to envision targeted therapies to congenital diseases. We have described here a novel disease-causing mutation in SMED-SL, which results in defective intracellular targeting and therefore loss of in vivo ligand induced phophorylation of the DDR2 protein.

\section{Conclusions}

This study documents the clinical findings and radiological features of two siblings with SMED-SL and a novel homozygous truncating mutation in the DDR2 gene. The novel mutation affects the intracellular trafficking of DDR2 and consequently collagen induced activation of the receptor. In addition, our findings illustrates that the C-terminal amino acids are crucial for the stability of the DDR2 protein, since the novel mutation leads to loss of the C-terminal 32 amino acids of the protein.

\section{Competing interests}

The authors declare no competing interests in the preparation or publication of the data in this manuscript.

\section{Authors' contributions}

$B R A, L A$ and $B L$ were responsible for the project conception and design of experiments. AJ performed genetic analysis, PK and HX performed confocal microscopy, biochemical assays and generated the figures. AAK, AAS, AG, MAA and LAA conducted clinical evaluations and compiled data. PK, LA, BRA, BL and HX wrote and edited the manuscript. All authors read and approved the final manuscript.

\section{Acknowledgements}

The authors would like to thank the patients' family for participating in this research. We are grateful to Mr. Saeed Tariq for helping with confocal microscopy imaging.

\section{Funding}

This work was supported by the United Arab Emirates University (Grant \# 31 M092) to B.R.A. and the Biotechnology and Biological Sciences Research Council, UK (Grant \# BB/I011226/1) to B.L.

\section{Author details}

'Department of Genetics, Sultan Qaboos University Hospital, Sultan Qaboos University, Al koudh, 123, Muscat, Sultanate of Oman. ${ }^{2}$ Department of Pathology, College of Medicine and Heath Sciences, United Arab Emirates University, P.O. Box 17666, Al Ain, United Arab Emirates. ${ }^{3}$ National Heart and Lung Institute, Imperial College London, London SW7 2AZ, United Kingdom. ${ }^{4}$ Pediatric Ophthalmology, Sultan Qaboos University Hospital, Sultan Qaboos University, Alkoudh, 123, Muscat, Sultanate of Oman. ${ }^{5}$ Department of Paediatrics, College of Medicine and Heath Sciences, United Arab Emirates University, Al Ain, United Arab Emirates.

Received: 16 March 2014 Accepted: 4 April 2014

Published: 11 April 2014

\section{References}

1. Borochowitz Z, Langer LO Jr, Gruber HE, Lachman R, Katznelson MB, Rimoin DL: Spondylo-meta-epiphyseal dysplasia (SMED), short limb-hand type: a congenital familial skeletal dysplasia with distinctive features and histopathology. Am J Med Genet 1993, 45:320-326.

2. Langer LO Jr, Wolfson BJ, Scott Cl Jr, Reid CS, Schidlow DV, Millar EA, Borns PF, Lubicky JP, Carpenter BL: Further delineation of spondylo-meta-epiphyseal dysplasia, short limb-abnormal calcification type, with emphasis on diagnostic features. Am J Med Genet 1993, 45:488-500.

3. Smithson SF, Grier D, Hall CM: Spondylo-meta-epiphyseal dysplasia, short limb-abnormal calcification type. Clin Dysmorphol 2009, 18:31-35.

4. Al-Gazali LI, Bakalinova D, Sztriha L: Spondylo-meta-epiphyseal dysplasia, short limb, abnormal calcification type. Clin Dysmorphol 1996, 5:197-206.

5. Dias C, Cairns R, Patel MS: Sudden death in spondylo-meta-epiphyseal dysplasia, short limb-abnormal calcification type. Clin Dysmorphol 2009, 18:25-29.

6. Bargal R, Cormier-Daire V, Ben-Neriah Z, Le Merrer M, Sosna J, Melki J, Zangen DH, Smithson SF, Borochowitz Z, Belostotsky R, Raas-Rothschild A: Mutations in DDR2 gene cause SMED with short limbs and abnormal calcifications. Am J Hum Genet 2009, 84:80-84.

7. Ali BR, Xu H, Akawi NA, John A, Karuvantevida NS, Langer R, Al-Gazali L, Leitinger B: Trafficking defects and loss of ligand binding are the underlying causes of all reported DDR2 missense mutations found in SMED-SL patients. Hum Mol Genet 2010, 19:2239-2250.

8. Vogel W, Gish GD, Alves F, Pawson T: The discoidin domain receptor tyrosine kinases are activated by collagen. Mol Cell 1997, 1:13-23.

9. Shrivastava A, Radziejewski C, Campbell E, Kovac L, McGlynn M, Ryan TE, Davis S, Goldfarb MP, Glass DJ, Lemke G, Yancopoulos GD: An orphan receptor tyrosine kinase family whose members serve as nonintegrin collagen receptors. Mol Cell 1997, 1:25-34.

10. Vogel WF, Abdulhussein R, Ford CE: Sensing extracellular matrix: an update on discoidin domain receptor function. Cell Signal 2006, 18:1108-1116. 
11. Leitinger B: Transmembrane collagen receptors. Annu Rev Cell Dev Biol 2011, 27:265-290.

12. Valiathan RR, Marco M, Leitinger B, Kleer CG, Fridman R: Discoidin domain receptor tyrosine kinases: new players in cancer progression. Cancer Metastasis Rev 2012, 31:295-321.

13. Leitinger B, Kwan AP: The discoidin domain receptor DDR2 is a receptor for type X collagen. Matrix Biol 2006, 25:355-364.

14. Xu L, Peng H, Wu D, Hu K, Goldring MB, Olsen BR, Li Y: Activation of the discoidin domain receptor 2 induces expression of matrix metalloproteinase 13 associated with osteoarthritis in mice. J Biol Chem 2005, 280:548-555.

15. Olaso E, Labrador JP, Wang L, Ikeda K, Eng FJ, Klein R, Lovett DH, Lin HC, Friedman SL: Discoidin domain receptor 2 regulates fibroblast proliferation and migration through the extracellular matrix in association with transcriptional activation of matrix metalloproteinase-2. J Biol Chem 2002 277:3606-3613.

16. Flynn LA, Blissett AR, Calomeni EP, Agarwal G: Inhibition of collagen fibrillogenesis by cells expressing soluble extracellular domains of DDR1 and DDR2. J Mol Biol 2010, 395:533-543.

17. Labrador JP, Azcoitia V, Tuckermann J, Lin C, Olaso E, Manes S, Bruckner K, Goergen JL, Lemke G, Yancopoulos G, Angel P, Martinez C, Klein R: The collagen receptor DDR2 regulates proliferation and its elimination leads to dwarfism. EMBO Rep 2001, 2:446-452.

18. Kano K, Marin de Evsikova C, Young J, Wnek C, Maddatu TP, Nishina PM, Naggert JK: A novel dwarfism with gonadal dysfunction due to loss-of-function allele of the collagen receptor gene, Ddr2, in the mouse. Mol Endocrinol 2008, 22:1866-1880.

19. Kano K, Kitamura A, Matsuwaki T, Morimatsu M, Naito K: Discoidin domain receptor 2 (DDR2) is required for maintenance of spermatogenesis in male mice. Mol Reprod Dev 2010, 77:29-37.

20. Matsumura H, Kano K, Marin De Evsikova C, Young JA, Nishina PM, Naggert $J \mathrm{~K}$, Naito K: Transcriptome analysis reveals an unexpected role of a collagen tyrosine kinase receptor gene, Ddr2, as a regulator of ovarian function. Physiol Genomics 2009, 39:120-129.

21. Carafoli F, Hohenester E: Collagen recognition and transmembrane signalling by discoidin domain receptors. Biochim Biophys Acta 1834 2013:2187-2194

22. Leitinger $\mathrm{B}$ : Molecular analysis of collagen binding by the human discoidin domain receptors, DDR1 and DDR2: identification of collagen binding sites in DDR2. J Biol Chem 2003, 278:16761-16769.

23. Carafoli F, Mayer MC, Shiraishi K, Pecheva MA, Chan LY, Nan R, Leitinger B, Hohenester E: Structure of the discoidin domain receptor 1 extracellular region bound to an inhibitory Fab fragment reveals features important for signaling. Structure 2012, 20:688-697.

24. Mihai C, Chotani M, Elton TS, Agarwal G: Mapping of DDR1 distribution and oligomerization on the cell surface by FRET microscopy. $J$ Mol Biol 2009, 385:432-445

25. Noordeen NA, Carafoli F, Hohenester E, Horton MA, Leitinger B: A transmembrane leucine zipper is required for activation of the dimeric receptor tyrosine kinase DDR1. J Biol Chem 2006, 281:22744-22751.

26. Carafoli F, Bihan D, Stathopoulos S, Konitsiotis AD, Kvansakul M, Farndale RW, Leitinger B, Hohenester E: Crystallographic insight into collagen recognition by discoidin domain receptor 2 . Structure 2009, 17:1573-1581.

27. Zhang Y, Su J, Yu J, Bu X, Ren T, Liu X, Yao L: An essential role of discoidin domain receptor 2 (DDR2) in osteoblast differentiation and chondrocyte maturation via modulation of Runx2 activation. J Bone Miner Res 2011, 26:604-617

28. Lin KL, Chou CH, Hsieh SC, Hwa SY, Lee MT, Wang FF: Transcriptional upregulation of DDR2 by ATF4 facilitates osteoblastic differentiation through p38 MAPK-mediated Runx2 activation. J Bone Miner Res 2010, 25:2489-2503

29. Khosravi R, Sodek KL, Faibish M, Trackman PC: Collagen advanced glycation inhibits its discoidin domain receptor 2 (DDR2)-mediated induction of lysyl oxidase in osteoblasts. Bone 2014, 58:33-41.

30. Gualeni B, Rajpar MH, Kellogg A, Bell PA, Arvan P, Boot-Handford RP, Briggs MD: A novel transgenic mouse model of growth plate dysplasia reveals that decreased chondrocyte proliferation due to chronic ER stress is a key factor in reduced bone growth. Dis Model Mech 2013, 6(6):1414-25.

31. Chen Y, Bellamy WP, Seabra MC, Field MC, Ali BR: ER-associated protein degradation is a common mechanism underpinning numerous monogenic diseases including robinow syndrome. Hum Mol Genet 2005, 14:2559-2569.
32. Ali BR, Jeffery S, Patel N, Tinworth LE, Meguid N, Patton MA, Afzal AR: Novel robinow syndrome causing mutations in the proximal region of the frizzled-like domain of ROR2 are retained in the endoplasmic reticulum. Hum Genet 2007, 122:389-395.

33. Hume AN, Buttgereit J, Al-Awadhi AM, Al-Suwaidi SS, John A, Bader M, Seabra MC, Al-Gazali L, Ali BR: Defective cellular trafficking of missense NPR-B mutants is the major mechanism underlying acromesomelic dysplasia-type maroteaux. Hum Mol Genet 2009, 18:267-277.

34. McCracken AA, Brodsky JL: Evolving questions and paradigm shifts in endoplasmic-reticulum-associated degradation (ERAD). Bioessays 2003, 25:868-877.

35. Aridor M, Hannan LA: Traffic jam: a compendium of human diseases that affect intracellular transport processes. Traffic 2000, 1:836-851.

36. Aridor M, Hannan LA: Traffic jams II: an update of diseases of intracellular transport. Traffic 2002, 3:781-790

37. Guerriero CJ, Brodsky JL: The delicate balance between secreted protein folding and endoplasmic reticulum-associated degradation in human physiology. Physiol Rev 2012, 92:537-576.

38. Holbrook JA, Neu-Yilik G, Hentze MW, Kulozik AE: Nonsense-mediated decay approaches the clinic. Nat Genet 2004, 36:801-808.

39. Konitsiotis AD, Raynal N, Bihan D, Hohenester E, Farndale RW, Leitinger B: Characterization of high affinity binding motifs for the discoidin domain receptor DDR2 in collagen. J Biol Chem 2008, 283:6861-6868.

40. Phan TN, Wong EL, Sun X, Kim G, Jung SH, Yoon CN, Yang BS: Low stability and a conserved $\mathrm{N}$-glycosylation site are associated with regulation of the discoidin domain receptor family by glucose via post-translational N-glycosylation. Biosci Biotechnol Biochem 2013, 77:1907-1916.

41. Mohan RR, Wilson SE: Discoidin domain receptor (DDR) 1 and 2: collagenactivated tyrosine kinase receptors in the cornea. Exp Eye Res 2001, 72:87-92.

42. Isidor B, Lindenbaum P, Pichon O, Bezieau S, Dina C, Jacquemont S, MartinCoignard D, Thauvin-Robinet C, Le Merrer M, Mandel JL, David A, Faivre L, Cormier-Daire $V$, Redon $R$, Le Caignec $C$ : Truncating mutations in the last exon of NOTCH2 cause a rare skeletal disorder with osteoporosis. Nat Genet 2011, 43:306-308.

43. Alves F, Saupe S, Ledwon M, Schaub F, Hiddemann W, Vogel WF: Identification of two novel, kinase-deficient variants of discoidin domain receptor 1: differential expression in human colon cancer cell lines. FASEB J 2001, 15:1321-1323.

44. Lemmon MA, Schlessinger J: Cell signaling by receptor tyrosine kinases. Cell 2010, 141:1117-1134

45. Leitinger B: Discoidin domain receptor functions in physiological and pathological conditions. Int Rev Cell Mol Biol 2014, 310:39-87.

46. Ali BR: Is cystic fibrosis-related diabetes an apoptotic consequence of ER stress in pancreatic cells? Med Hypotheses 2009, 72:55-57.

doi:10.1186/1471-2350-15-42

Cite this article as: Al-Kindi et al:: A novel mutation in DDR2 causing spondylo-meta-epiphyseal dysplasia with short limbs and abnormal calcifications (SMED-SL) results in defective intra-cellular trafficking. BMC Medical Genetics 2014 15:42.

\section{Submit your next manuscript to BioMed Central and take full advantage of:}

- Convenient online submission

- Thorough peer review

- No space constraints or color figure charges

- Immediate publication on acceptance

- Inclusion in PubMed, CAS, Scopus and Google Scholar

- Research which is freely available for redistribution 